\title{
Palinologia de espécies de Malpighiaceae Juss. ocorrentes nas restingas do Estado do Rio de Janeiro
}

\author{
Vania Gonçalves-Esteves ${ }^{1,2}$, Edio Ferreira Soares Junior ${ }^{1,2}$ e Cláudia Barbieri Ferreira Mendonça ${ }^{1,2}$
}

Recebido: 26.04.2007; aceito: 06.11.2007

\begin{abstract}
Palynology of species of Malpighiaceae Juss. that occurs in "restingas" of Rio de Janeiro State). It were analyzed 17 species subordinate to eight genera that occur in Rio de Janeiro "restingas", aiming to contribute to the pollinic characterization of this species and to the pollinic catalog of "restingas" of Rio de Janeiro. The pollen grains were acetolysed, measured, described and illustrated under light microscopy to observe details of surface, non acetolysed pollen grains were analysed under SEM. Only Byrsonima sericea has small, tricolporate and isopolar pollen grains. On other species were apolar pollen grains and presented characteristics that were used in the pollinic key. The main characteristics employed were size, number and dimension of the apertures, sexine and colpoid ornamentation. It conclude that analyzed species were palylonologically distinct and the pollinic characteristics were considered to be a good available resource to the researchers that will be able to use in the Taxonomy and in other related areas.
\end{abstract}

Key words: Malpighiaceae, palynology, "restingas", Rio de Janeiro

RESUMO - (Palinologia de espécies de Malpighiaceae Juss. ocorrentes nas restingas do Estado do Rio de Janeiro). Foram analisadas 17 espécies subordinadas a oito gêneros ocorrentes nas restingas do Estado do Rio de Janeiro, com o objetivo de contribuir para a caracterização polínica dessas espécies e para o catálogo polínico das restingas do Rio de Janeiro. Os grãos de pólen foram acetolisados, analisados e fotomicrografados sob microscopia de luz. Para observar detalhes da superfície, grãos de pólen não acetolisados foram analisados em MEV. Apenas Byrsonima sericea possui grãos de pólen isopolares, pequenos e tricolporados. Nas demais espécies os grãos de pólen foram apolares e apresentaram atributos que foram utilizados na chave polínica sendo as principais caraterísticas utilizadas, o tamanho dos grãos de pólen, a quantidade e dimensões dos poros, a ornamentação da sexina e dos colpóides. Conclui-se que as espécies são palinologicamente distintas e as características polínicas mostraram ser um bom recurso disponível aos pesquisadores que poderão utilizar na taxonomia e demais áreas afins.

Palavras-chave: Malpighiaceae, palinologia, restingas, Rio de Janeiro

\section{Introdução}

Malpighiaceae Juss. pertence à ordem Malpighiales, com cerca de 63 gêneros e, aproximadamente, 600 espécies pantropicais. É uma família distinta por suas folhas opostas, inteiras, com pares de estípulas; tricomas unicelulares em forma de $\mathrm{T}$, quase sempre de cor marrom, sendo freqüente a presença de glândulas achatadas conspícuas sobre o limbo foliar ou o pecíolo. As flores com 4 ou 5 sépalas, freqüentemente, pareadas por glândulas, as pétalas são fortemente clavadas e dobradas no botão, com 3 carpelos, cada um com um único óvulo. As sépalas e os filetes são freqüentemente persistentes na base do fruto, que é semelhante a uma sâmara com um variável número de alas (APG 2003).

De acordo com dados do APG (2003), a família Malpighiaceae está dividida em duas subfamílias: Malpighioideae Burnett, de ocorrência tropical e subtropical, e Byrsonimoideae W.R. Anderson, com predomínio na América tropical. Malpighioideae caracteriza-se por apresentar grãos de pólen "globais", simétricos, 4 a poliporados, estilete variável, estigma terminal, fruto alado ou não, cromossomo $n=(9)-10$. Com sete gêneros e cerca de 560 espécies, a subfamília predomina nas regiões tropicais e subtropicais, especialmente nas Américas. Byrsonimoideae ocorre na América tropical, diferencia-se de Malpighioideae por possuir laticíferos articulados, estilete subulado, estigma terminal. Byrsonima é o único gênero da

1. Museu Nacional, UFRJ, Departamento de Botânica, Quinta da Boa Vista, São Cristóvão, 20940-040 Rio de Janeiro, RJ, Brasil

2. Autor para correspondência: esteves.vr@gmail.com 
subfamília e possui cerca de 150 espécies, todas ocorrentes na América tropical.

A filogenia da família vem sendo analisada em vários trabalhos através do seqüenciamento genético e estudos citogenéticos de várias espécies relacionando o número de cromossomos à diversidade morfológica de gêneros, como: Banisteriopsis Rob. e Heteropterys Kunth (Lombello \& Forni 2001). São estudados o "murici" (Byrsonima crassifolia L. Rich), com a pesquisa de suas essências (Alves \& Franco 2003), e a "acerola" (Malpighia emarginata DC.), com o isolamento de suas enzimas, sem falar no uso da polpa de ambos, para a indústria alimentícia. Estas atividades revelam uma pequena parcela da importância econômica da família Malpighiaceae.

Pode-se citar ainda, os estudos de algumas espécies dos gêneros Heteropterys Kunth, Banisteriopsis Rob. ex Small e Malpighia L. em pesquisas de fármacos para o combate ao câncer e ao Mal de Parkinson (Schwarz et al. 2003), enquanto outros estudos investigam o grau de toxidez e atividade antiviral de espécies do gênero Tetrapterys Cav. ou, ainda, qualidades antibióticas de Byrsonima crassifolia (Melo et al. 2001).

A família Malpighiaceae está representada em inúmeros levantamentos florísticos, os quais auxiliam na análise dos aspectos ecológicos de insetos polinizadores, principalmente, devido à coleta de óleo dos elaióforos de suas flores (Aguiar \& De-Almeida 2002).

Estudos palinológicos na família foram realizados por Erdtman (1952), Salgado-Labouriau (1973), Makino (1986), Barth (1989) e Roubik \& Moreno (1991).

Pretende-se, com esse trabalho, acrescentar informações que auxiliem a taxonomia da família Malpighiaceae, através do estudo palinológico de 17 espécies subordinadas a oito gêneros ocorrentes nas restingas do Estado do Rio de Janeiro.

\section{Material e métodos}

O material botânico utilizado foi proveniente de botões florais bem desenvolvidos, retirados de exsicatas dos herbários: Herbarium Bradeanum (HB), do Museu Nacional/UFRJ (R), Alberto Castelhanos/FEEMA (GUA) e do Instituto de Pesquisas do Jardim Botânico do Rio de Janeiro (RB).

Foi estudado um total de 17 espécies, provenientes das restingas do Estado do Rio de Janeiro.

Segue a relação do material estudado:
Banisteriopsis sellowiana (A. Juss.) B. Gates BRASIL. Rio DE JANEIRO: Maricá, restinga de Maricá, IV-1988, J. Fontella \& B. Rapoport s.n. * (R194510); Saquarema, Praia de Itaúna, restinga, 28-IX-1988, D. Araújo et al. 8598 (GUA); Rio de Janeiro, restinga de Jacarepaguá, 4-V-1951, E. Pereira et al. 4363 (RB). Byrsonima sericea DC. - Rio DE JANEIRO: Arraial do Cabo, 29-I-1950, C.A.M. Weiss s.n. (RB75761); Rio de Janeiro, Bosque da Barra, restinga de Jacarepaguá, 26-IX-1982, M.H.O. Lemos s.n.* (RB224216); Rio de Janeiro, Estrada da Restinga de Marambaia, km 2, 20-X-1966, E. Guimarães \& D. Sucre 66 (RB); BAHIA: Salvador, restinga da Praia de Itacimirim, 27-X-1989, H. Passinho \& M.V. Campos s.n. (RB319120). Heteropterys alternifolia W.R. Anderson - RIO DE JANEIRO: Saquarema, Reserva Ecológica de Jacarepiá, 28-IX-1989, D. Araújo 9959* (GUA). Heteropterys bicolor A. Juss. - RIO DE JANEIRO: Arraial do Cabo, Estrada antiga para Búzios, D. Araújo et al. 10303* (GUA43223); Cabo Frio, Morro do Gavião, 20-X-1966, D. Sucre 3933 (RB). Heteropterys chrysophylla (Lam.) Kunth - RIO DE JANeIro: Macaé, entre Quissamã e Lagoa Feia, restinga, 2-III-1965, H. Sick s.n.* (HB40740); Rio das Ostras, Praia Virgem, 14-III-2000, H.N. Braga 904 (RB); Rio de Janeiro, Barra da Tijuca, 6-I-1946, G. Hatschbach 9785 (RB). Heteropterys coleoptera A. Juss. - Rio DE JANEIRo: Carapebus, restinga de Carapebus, 6-X-1997, F.C. Ferreira 10 (R); Carapebus, próximo Fazenda São Lázaro, 20-VIII-1997, I.M. Silva et al. s.n. (R191987); Restinga do Recreio dos Bandeirantes, 28-I-1967, E. Pereira s.n.* (HB44804); idem, Carapebus, Restinga de Carapebus, 20-XI-1987, D. Araújo \& N.G. Maciel 3235 (GUA). Heteropterys fluminensis (Griseb.) W. Anderson - Rio DE JANEIRO: Restinga de Grumari, 28-IX-1989, D. Araújo 9071* (GUA); Jacarepaguá, Represa do Camorim, 1-X-1958, E. Pereira \& D. Sucre 4394 (RB). Heteropterys rufula A. Juss. - Rio DE JANEIRO: Barra da Tijuca, Restinga de Itapeba, 30-II-1978, M.C. Vianna 233* (GUA); idem, Rio de Janeiro, Dunas de Jacarepaguá, s.d., A. Castelhanos 24921 (GUA). Hiraea cuneata Griseb. - Rio DE JANEIRO: Carapebus, Restinga de Carapebus, 10-XII-1996, R. Moura et al. 28*(R). Peixotoa hispidula A. Juss. - Rio DE JANEIRO: Carapebus, Restinga de Carapebus, 21-VI-1996, V.L.C. Martins 327 et al.* (R); Carapebus, próximo da Fazenda São Lázaro, 21-VI-1995, M.G. Santos et al. 359 (GUA); Macaé, Restinga de Carapebus, 
16-III-1995, V. Esteves et al. 976 (R). Stigmaphyllon arenicola C. Anderson - Rio DE JANEIRO: Angra dos Reis, Ilha Grande, Reserva Biológica da Praia do Sul, 20-XI-1987, D. Araújo et al. 7305* (GUA); Restinga de Jacarepaguá, Pedra de Itaúna, 24-IV-1973, E.F. Guimarães s.n. (RB166013); Restinga de Jacarepaguá, 7-V-1958, A.P. Duarte \& E. Pereira s.n. (RB109944). Stigmaphyllon auriculatum (Cav.) A. Juss. - Rio de JANEIRO: Búzios, Serra das Emerenças, 26-III-1987, A. Quinet \& F.F. Morais 647 (RB); Rio de Janeiro, formação rupícula do Recreio dos Bandeirantes, 20-IX-1961, D. Sucre 7935* (RB); Rio de Janeiro, APA da Praia Vermelha, ao lado do morro do Pão de Açúcar, 25-III-1987, A. Rodrigues 76 (RB); Ilha de Paquetá, 9-V-1988, H.M. Ferreira \& C.L.B. de Abreu s.n. (RB284162). Stigmaphyllon ciliatum (Lam.) A. Juss. - Macaé, junto à Lagoa Paulista, 1-X-1997, D. Araújo \& M.C. Maciel 4688* (GUA). Stigmaphyllon paralias A. Juss. - BAHIA: Santa Cruz Cabrália, 18-III-1974, R.M. Harley 17124 (RB); Rio DE JANEIRo, Restinga de Jacarepaguá, 5-XI-1958, E. Pereira, D. Sucre \& Duarte s.n.* (RB109194); Maricá, Barra de Maricá, 28-XI-1988, A. Souza \& H. Pereira 2551 (R). Tetrapterys acutifolia Griseb. - RIO DE JANEIRO: Macaé, Restinga de Cabiúnas, 20-XI-1987, D. Araújo \& N.C. Maciel 7077* (GUA); Restinga de Jurubatiba, 21-III-1999 D. Araújo \& O. Peixoto 6527 (GUA); Macaé, Fazenda São Lázaro, próximo ao Canal Macaé, Campos, 20-XII-1989, D. Araújo 10206 (GUA); Niterói, Restinga de Itaipú, 20-XI-1985, D. Araújo 3205 (GUA). Tetrapterys phlomoides (Spreng.) Nied. - Rio DE JANEIRO: Angra dos Reis, Ilha Grande, Reserva Biológica Estrada da Praia do Sul, 20-XI-1987, R. Ribeiro 1894* (GUA); Macaé, Restinga de Macaé, loteamento Lagomar, 2-XII-1994, C. Farney \& D. Araújo 3466 (GUA). Thryallis brachystachys (Lindl.) Kuntze - RIO DE JANEIRO: Saquarema, Restinga de Ipitangas, 24-III-1987, C. Farney et al. 1371* (RB); Campos dos Goytacazes, Fazenda Canaã, IX-1976, Sampaio 7893 (R); BAHIA: Jequié, transição para a Caatinga, 22-I-1965, R.P. Belém \& J.M. Mendes s.n. (RB140523).

Para análise em microscópio de luz, os grãos de pólen foram tratados pelo método acetolítico (Erdtman 1952) com modificações propostas por Melhem et al. (2003). Para cada espécie analisada foram estudados os grãos de pólen de, no máximo quatro espécimens, um padrão (assinalado com um asterisco após o nome do coletor) e três comparações.
As lâminas com o material polínico encontramse depositadas na Palinoteca do Laboratório de Palinologia Álvaro Xavier Moreira do Departamento de Botânica do Museu Nacional/UFRJ, Rio de Janeiro.

Todas as medidas foram obtidas no prazo máximo de sete dias após a acetólise, a fím de evitar alterações nas dimensões dos grãos de pólen (SalgadoLabouriau 1973).

Do material padrão foram mensurados dois diâmetros em grãos de pólen apolares; nos grãos de pólen isopolares, foram medidos, em vista equatorial o diâmetro polar e o equatorial. Foram tomadas, aleatoriamente, 25 medidas dos diâmetros, distribuídas em um mínimo de três lâminas, de modo a uniformizar a amostra (Salgado-Labouriau et al. 1965). Foram calculados a média aritmética $(\bar{x})$; o desvio padrão da média $\left(\mathrm{s}_{\overline{\mathrm{x}}}\right)$; o coeficiente de variabilidade $(\mathrm{CV} \%)$ e o intervalo de confiança a $95 \%$ (I.C).

Foram realizadas 10 medidas e calculada a média aritmética da espessura da exina, das dimensões das aberturas, dos diâmetros dos grãos de pólen apolares e isopolares do material de comparação.

As medidas e as fotomicrografias foram realizadas em microscópio de luz Zeiss com objetivas em aumentos de 40X e 100X.

A partir dos resultados obtidos no presente estudo, foram consideradas, para os poros, as seguintes classes de tamanho: pequenos $(2,0-3,0 \mu \mathrm{m})$, médios $(4,7-6,0 \mu \mathrm{m})$ ou grandes $(6,5-7,5 \mu \mathrm{m})$.

A terminologia adotada e as descrições polínicas seguiram os critérios de Barth \& Melhem (1988) e Punt et al. (2007), levando-se em consideração o tamanho, a forma, o número de aberturas e o padrão da ornamentação da sexina.

\section{Resultados}

Banisteriopsis sellowiana - (figuras 1-4) grãos de pólen médios (tabelas 1,4), apolares, cuboidais, 6-porados, com colpóides e sexina rugulada, com regiões psiladas.

Aberturas: são poros médios (tabela 2), com margem espessa. Os colpóides estão dispersos sobre o grão de pólen (figuras 2, 3), podendo ou não estar associados aos poros, possuem superfície granulada e são pouco profundos (figuras 3, 4).

Exina: nexina mais espessa do que a sexina, formada por rúgulas conspícuas e regiões psiladas próximas aos colpóides, presença de perfurações esparsas (figuras 1, 3, 4). 
Tabela 1. Medidas $(\mu \mathrm{m})$ dos diâmetros dos grãos de pólen de espécies de Malpighiaceae. Média aritmética ( $\bar{x})$, desvio padrão da média $(\mathrm{s} \bar{x})$, intervalo de confiança (I.C.) $(\mathrm{n}=25)$.

\begin{tabular}{|c|c|c|c|c|c|c|}
\hline \multirow[b]{2}{*}{ Espécie } & \multicolumn{3}{|c|}{ Diâmetro I } & \multicolumn{3}{|c|}{ Diâmetro II } \\
\hline & Faixa de variação & $\bar{x} \pm s_{\bar{x}}$ & I.C. $95 \%$ & Faixa de variação & $\bar{x} \pm s_{\bar{x}}$ & I.C. $95 \%$ \\
\hline Banisteriopsis sellowiana & $37,8-40,5$ & $38,9 \pm 0,2$ & $38,5-39,3$ & $37,8-41,4$ & $39,5 \pm 0,2$ & $39,1-39,9$ \\
\hline Heteropteris alternifolia & $42,3-45,0$ & $44,4 \pm 0,2$ & $44,0-44,8$ & $45,0-45,9$ & $45,0 \pm 0,1$ & $44,9-45,1$ \\
\hline H. bicolor & $45,0-50,4$ & $48,2 \pm 0,3$ & $47,6-48,8$ & $48,6-51,3$ & $49,9 \pm 0,2$ & $49,5-50,3$ \\
\hline H. chrysophylla & $51,7-54,0$ & $53,4 \pm 0,2$ & $53,0-53,8$ & $52,2-54,0$ & $53,8 \pm 0,1$ & $53,5-54,1$ \\
\hline H. coleoptera & $43,2-45,9$ & $45,0 \pm 0,1$ & $44,8-45,2$ & $45,0-46,8$ & $45,2 \pm 0,1$ & $45,0-45,4$ \\
\hline H. fluminensis & $36,0-37,8$ & $36,5 \pm 0,1$ & $36,3-36,7$ & $36,0-37,8$ & $36,7 \pm 0,2$ & $36,4-37,0$ \\
\hline H. rufula & $34,2-36,0$ & $35,2 \pm 0,2$ & $34.8-35,6$ & $35,1-36,9$ & $36,1 \pm 0,1$ & $35,8-36.4$ \\
\hline Hiraea cuneata & $36,0-40,5$ & $37,7 \pm 0,2$ & $37,3-38,1$ & $37,8-40,5$ & $39,0 \pm 0,2$ & $38,6-39,4$ \\
\hline Peixotoa hispidula & $44,1-45,9$ & $45,5 \pm 0,2$ & $45,1-46,9$ & $45,0-50,4$ & $46,3 \pm 0,2$ & $45,9-46,7$ \\
\hline Stigmaphyllon arenicola & $56,7-61,2$ & $59,1 \pm 0,3$ & $58,5-59,7$ & $58,6-63,0$ & $60,5 \pm 0,3$ & $59,9-61,1$ \\
\hline S. auriculatum & $40,5-41,4$ & $41,3 \pm 0,1$ & $41,1-41,5$ & $41,4-42,3$ & $42,1 \pm 0,1$ & $41,9-42,3$ \\
\hline S. ciliatum & $48,6-50,4$ & $49,6 \pm 0,2$ & $49,2-50,0$ & $49,5-50,4$ & $50,7 \pm 0,1$ & $50,4-51,0$ \\
\hline S. paralias & $51,3-54,0$ & $53,1 \pm 0,3$ & $52,5-53,7$ & $54,0-55,8$ & $54,6 \pm 0,2$ & $54,2-55,0$ \\
\hline Tetrapteris acutifolia & $31,5-35,1$ & $33,2 \pm 0,2$ & $32,8-33,6$ & $33,3-36,0$ & $34,3 \pm 0,2$ & $33,9-34,7$ \\
\hline T. phlomoides & $45,1-45,9$ & $45,1 \pm 0,1$ & $44,9-45,3$ & $45,0-46,8$ & $45,9 \pm 0,2$ & $45,6-46,2$ \\
\hline Thryallis brachystachys & $26,5-29,3$ & $27,9 \pm 0,1$ & $27,0-28,9$ & $27,5-29,9$ & $28,7 \pm 0,1$ & $27,9-29,7$ \\
\hline
\end{tabular}

Byrsonima sericea - (figuras 5-8) grãos de pólen pequenos, isopolares, área polar pequena (IAP $=0,43 \mu \mathrm{m})$, âmbito subcircular, prolatoesferoidais, 3-colporados, sexina microrreticulada.

Aberturas: são cólporos, formados por colpos longos $(11,9 \times 2,0 \mu \mathrm{m})$, largos, endoabertura lalongada $(2,1 \times 6,7 \mu \mathrm{m})$ com extremidades arredondadas (figura 7).

Exina: ca. 2,0 $\mu \mathrm{m}$; sexina $(1,0 \mu \mathrm{m})$ tão espessa quanto a nexina $(1,0 \mu \mathrm{m})$, formada por microrretículos (figuras 6,8).

Medidas $(\mu \mathrm{m})$ : vista equatorial - Diâmetro polar $=(16,0-18,0) 17,0 \pm 0,2 ;$ I.C. $95 \%=16,7-17,3$; Diâmetro equatorial $=(15,0-17,0) 16,3 \pm 0,1 ;$ I.C. $95 \%$ $=16,1-16,5$. Vista polar - Diâmetro equatorial $=$ $(16,0-17,0) 16,7 ;$ lado do apocolpo $=(6,3-7,5) 7,1$.

A tabela 3 mostra que os resultados encontrados nos espécimes de comparação apresentam os valores dos diâmetros polar e equatorial, na maioria, dentro dos limites da faixa de variação quando comparados com o material padrão. A forma se manteve a mesma do material padrão.

\section{Heteropterys (figuras 9-34)}

H. alternifolia (figuras 9-13), H. bicolor (figuras 14-18), H. chrysophylla (figuras 19-22), $H$. coleoptera (figuras 23-27), H. fluminensis (figuras 28-31), H. rufula (figuras 32-34).
Grãos de pólen médios, grandes apenas em H. chrysophylla (tabelas 1, 4), apolares, subesferoidais, 6-8-porados, com colpóides, sexina rugulada, perfurada ou escabrada.

Aberturas: poros médios, grandes apenas em H. chrysophylla (tabela 2), margem espessa; os colpóidessão nítidos e estão sempre associados aos poros, possuem superfície variavelmente granulada (figuras 13, 17, 21, 25). Em $H$. fluminensis, os colpóides são de difícil visualização (figuras 29, 31).

Exina: sexina formada por rúgulas distribuídas uniformemente pela superfície, com perfurações esparsas e sem área psilada em $H$. alternifólia (figuras 12, 13), $H$. chrysophylla (figuras 21, 22); rugulada com grandes áreas psiladas e perfurações esparsas em $H$. bicolor (figuras 17, 18); tectadoperfurada em $H$. coleoptera (figuras 25, 27); escabrada com perfurações em $H$. fluminensis (figuras 29, 31) e $H$. rufula (figuras 33, 34). Sexina mais espessa do que a nexina ou tão espessa quanto a nexina apenas em $H$. alternifolia (tabela 2).

Hiraea cuneata - (figuras 35-37) grãos de pólen médios, (tabelas 1, 4), apolares, subesferoidais, 6-porados, com colpóides, sexina rugulada.

Aberturas: poros médios (tabela 2), com margem espessa. Os colpóides são nítidos, com superfície granulada (figuras 35), estando ou não associados aos poros (figuras 36). 
Tabela 2. Média $(\mu \mathrm{m})$ das aberturas e da exina dos grãos de pólen de espécies de Malpighiaceae $(\mathrm{n}=10)$.

\begin{tabular}{|c|c|c|c|c|c|c|}
\hline & \multirow{2}{*}{$\begin{array}{c}\text { Poros } \\
\text { diâmetro }\end{array}$} & \multicolumn{5}{|c|}{ Exina } \\
\hline & & exina & sexina & nexina 1 & nexina 2 & nexina total \\
\hline Banisteriopsis sellowiana & 5,4 & 3,0 & 1,0 & 1,0 & 1,0 & 2,0 \\
\hline Heteropterys alternifolia & 4,7 & 3,9 & 1,9 & 1,0 & 1,0 & 2,0 \\
\hline H. bicolor & 5,2 & 7,1 & 5,0 & 1,0 & 1,0 & 2,0 \\
\hline H. chrysophylla & 7,3 & 5,6 & 3,6 & 1,0 & 1,0 & 2,0 \\
\hline H. coleoptera & 5,8 & 6,0 & 4,0 & 1,0 & 1,0 & 2,0 \\
\hline H. fluminensis & 5,9 & 3,0 & 2,0 & 0,5 & 0,5 & 1,0 \\
\hline H. rufula & 6,0 & 4,9 & 2,9 & 1,0 & 1,0 & 2,0 \\
\hline Hiraea cuneata & 5,0 & 5,0 & 3,0 & 1,0 & 1,0 & 2,0 \\
\hline Peixotoa hispidula & 7,5 & 5,0 & 3,0 & 1,0 & 1,0 & 2,0 \\
\hline Stigmaphyllon arenicola & 6,7 & 8,2 & 6,2 & 1,0 & 1,0 & 2,0 \\
\hline S. auriculatum & 7,4 & 5,0 & 3,0 & 1,0 & 1,0 & 2,0 \\
\hline S. ciliatum & 7,2 & 6,0 & 4,0 & 1,0 & 1,0 & 2,0 \\
\hline S. paralias & 5,9 & 5,5 & 3,9 & 1,0 & 0,7 & 1,7 \\
\hline Tetrapterys acutifolia & 4,9 & 4,0 & 2,0 & 1,0 & 1,0 & 2,0 \\
\hline T. phlomoides & 6,5 & 7,0 & 5,0 & 1,0 & 1,0 & 2,0 \\
\hline Thryallis brachystachys & 2,8 & 7,2 & 5,1 & 1,2 & 1,0 & 2,2 \\
\hline
\end{tabular}

Exina: sexina mais espessa do que a nexina (tabela 2), formada por rúgulas conspícuas, com áreas psiladas e perfurações esparsas próximo aos colpóides (figuras 36).

Peixotoa hispidula - (figuras 38-41) grãos de pólen médios, (tabelas 1, 4), apolares, cuboidais, 8-porados, com colpóides, sexina perfurada.

Aberturas: poros (tabela 2) grandes de margem espessa. Os colpóides são nítidos, com superfície granulada, associados ou não aos poros (figura 40).

Exina: sexina mais espessa do que a nexina (tabela 2), perfurada, com pequenas regiões ruguladas delimitadas pelos colpóides (figuras 38, 40, 41).

Stigmaphyllon (figuras 42-56)

S. arenicola (figuras 42-45), S. auriculatum (figuras 46-47), S. ciliatum (figuras 48-51), S. paralias (figuras 52-56).

Grãos de pólen médios ou grandes em S. arenicola e $S$. paralias, apenas médios em $S$. auriculatum e apenas grandes em $S$. ciliatum (tabelas 1, 4), apolares, subesferoidais, 6-8-10-porados, com colpóides, sexina rugulada ou rugulado-pilada.

Aberturas: são poros médios (tabela 2) em S. paralias ou grandes em $S$. arenicola, S. auriculatum e $S$. ciliatum (tabela 2), margem espessa ou levemente espessa apenas em $S$. paralias, 6 a 8 poros em $S$. auriculatum, 6-porados apenas em
S. paralias ou 10-porados apenas em S. arenicola e S. ciliatum. Os colpóides estão ou não associados aos poros, são nítidos, na maioria das espécies ou de difícil visualização apenas em $S$. paralias (figuras 52, 55); superfície granulada, com poucos grânulos em $S$. arenicola, com grânulos mais conspícuos em S. ciliatum (figura 51) e $S$. paralias (figuras 52, 55).

Exina: sexina mais espessa do que a nexina (tabela 2), com rúgulas em concavidades delimitadas por áreas psiladas largas e espessas em $S$. arenicola (figura 44), S. auriculatum (figura 47) e S. ciliatum (figura 51). Em S. paralias, a sexina possui rúgulas conspícuas, com inúmeros pilos de diferentes diâmetros entre os muros das rúgulas (figura 56).

\section{Tetrapterys (figuras 57-65)}

T. acutifolia (figuras 57-60), T. phlomoides (figuras 61-65).

Grãos de pólen médios, apolares, (tabelas 1, 4), subesferoidais, 6-porados, com colpóides, sexina microrreticulada com perfurações ou rugulada.

Aberturas: poros médios (tabela 2) em T. acutifolia e grandes em T. phlomoides, de margem espessa. Colpóides associados aos poros, colpóides nítidos, com grânulos apenas em T. phlomoides e delimitados por uma área psilada espessa (figuras 62, 64).

Exina: sexina é mais espessa que a nexina em T. phlomoides ou tão espessa quanto a nexina em 
T. acutifólia (tabela 2). Sexina microrreticulada com perfurações em T. acutifolia (figuras 58) ou rugulada, com áreas psiladas espessas em T. phlomoides (figuras 65).

Thryallis brachystachys (figuras 66-69) - grãos de pólen médios (tabelas 1, 4), apolares, subesferoidais, 6-porados, com colpóides, sexina insulada.

Aberturas: poros pequenos (tabela 2), de margem espessa. Os colpóides são pouco nítidos, pouco associados aos poros, com grânulos conspícuos.

Exina: sexina é mais espessa que a nexina (tabela 2). Sexina insulada, com perfurações esparsas.

A tabela 4 mostra os resultados encontrados nos espécimes de comparação dos táxons apolares. Os valores dos diâmetros I e II, na maioria dos espécimes, ficaram fora dos limites da faixa de variação quando comparados com o respectivo material padrão.

Tabela 3. Médias ( $\mu \mathrm{m})$ dos grãos de pólen do material de comparação, em vista equatorial, de Byrsonima sericea $(\mathrm{n}=10)$.

\begin{tabular}{lccc}
\hline Espécie & Diâmetro polar & Diâmetro equatorial & Forma \\
\hline Byrsonima sericea & & & prolato-esferoidal \\
C.A.M. Weiss & 17,0 & 15,9 & prolato-esferoidal \\
E. Guimarães \& D. Sucre 66 & 17,0 & 15,9 & prolato-esferoidal \\
H. Passarinho \& M.V. Campos & 18,9 & 18,6 & \\
\hline
\end{tabular}

Chave polínica para separação de espécies de Malpighiaceae

1. Grãos de pólen pequenos $(<25 \mu \mathrm{m})$, isopolares, 3 -colporados

Byrsonima sericea

1. Grãos de pólen médios ou grandes ( $>25 \mu \mathrm{m})$, apolares, porados, com colpóides

2. Grãos de pólen 10-porados

Stigmaphyllon arenicola,

Stigmaphyllon ciliatum

2. Grãos de pólen 6-8-porados

3. Grãos de pólen cuboidais

4. Grãos de pólen 6-porados, poros de tamanho médio, sexina rugulada ..... Banisteriopsis sellowiana

4. Grãos de pólen 8-porados, poros de tamanho grande, sexina perfurada

Peixotoa hispidula

3. Grãos de pólen subesferoidais

5. Sexina microrreticulada ou insulada

6. Sexina microrreticulada com perfurações e poros de tamanho médio.... Tetrapterys acutifolia

6. Sexina insulada, sem perfurações e poros de tamanho pequeno

Thryallis brachystachys

5. Sexina escabrada, tectado-perfurada ou rugulada

7. Sexina escabrada com perfurações

8. Colpóide de difícil visualização

Heteropterys fluminensis

8. Colpóides nítidos com superfície granulada

Heteropterys rufula

7. Sexina tectado-perfurada ou rugulada

9. Sexina tectado-perfurada.

Heteropterys coleoptera

9. Sexina rugulada

10. Sexina com concavidades delimitadas por áreas psiladas largas e espessas

Stigmaphyllon auriculatum

10. Sexina sem concavidades

11. Sexina com regiões psiladas

12. Poros de tamanho grande

Tetrapterys phlomoides

12. Poros de tamanho médio

13. Grãos de pólen de tamanho $>45,0 \mu \mathrm{m}$

Heteropterys bicolor

13. Grãos de pólen de tamanho $<41,5 \mu \mathrm{m}$ Hiraea cuneata

11. Sexina sem regiões psiladas 
14. Poros de tamanho grande, rúgulas inconspícuas ..... Heteropterys chrysophylla

14. Poros de tamanho médio, rúgulas conspícuas

15. Sexina sem pilos entre os muros das rúgulas, I.C. $95 \%(\mathrm{DI})=$ $44,0-44,8 \mu \mathrm{m}$ Heteropterys alternifolia

15. Sexina com inúmeros pilos de diferentes diâmetros entre os muros das rúgulas, I.C. $95 \%(\mathrm{DI})=52,5-53,7 \mu \mathrm{m}$ Stigmaphyllon paralias

Tabela 4. Médias ( $\mu \mathrm{m})$ dos grãos de pólen apolares do material de comparação de espécies de Malpighiaceae.

\begin{tabular}{|c|c|c|}
\hline Táxons & Diâmetro I & Diâmetro II \\
\hline \multicolumn{3}{|l|}{ Banisteriopsis sellowiana } \\
\hline D. Araújo et al. 8598 & 44,9 & 45,5 \\
\hline E. Pereira 4363 & 39,4 & 40,0 \\
\hline \multicolumn{3}{|l|}{ Heteropterys bicolor } \\
\hline D. Sucre 3933 & 45,6 & 47,6 \\
\hline \multicolumn{3}{|l|}{ Heteropterys chrysophylla } \\
\hline H.N. Braga 904 & 44,8 & 45,5 \\
\hline G. Hatschbach 9785 & 53,1 & 53,7 \\
\hline \multicolumn{3}{|l|}{ Heteropterys coleoptera } \\
\hline D. Araújo \& N.A. Maciel & 44,7 & 45,3 \\
\hline F. C. Ferreira 10 & 44,8 & 45,2 \\
\hline I.M. da Silva s.n. (R191987) & 44,9 & 45,3 \\
\hline \multicolumn{3}{|l|}{ Heteropterys fluminensis } \\
\hline E. Pereira 4394 & 37,4 & 38,2 \\
\hline \multicolumn{3}{|l|}{ Heteropterys rufula } \\
\hline A. Castelhanos 24921 & 27,1 & 27,8 \\
\hline \multicolumn{3}{|l|}{ Peixotoa hispidula A. Juss. } \\
\hline M.G. Santos et al. 359 & 46,2 & 47,1 \\
\hline V. Esteves et al. 976 & 45,7 & 46,5 \\
\hline \multicolumn{3}{|l|}{ Stigmaphyllon arenicola } \\
\hline E.F. Guimarães s.n. (RB166013) & 47,1 & 48,2 \\
\hline $\begin{array}{l}\text { A.P. Duarte \& E. Pereira s.n. } \\
\text { (RB109944) }\end{array}$ & 44,0 & 44,9 \\
\hline \multicolumn{3}{|l|}{ Stigmaphyllon auriculatum } \\
\hline A. Quinet \& F.F. Morais 647 & 35,6 & 36,0 \\
\hline A. Rodrigues 76 & 35,1 & 36,1 \\
\hline H.M. Ferreira \& C.L.B. de Abreu & 44,3 & 50,2 \\
\hline \multicolumn{3}{|l|}{ Stigmaphyllon paralias } \\
\hline A. Souza \& H. Pereira 2551 & 45,6 & 46,6 \\
\hline R.M. Harley 17124 & 37,7 & 38,3 \\
\hline \multicolumn{3}{|l|}{ Tetrapterys acutifolia } \\
\hline D. Araújo 3205 & 27,0 & 27,3 \\
\hline D. Araújo 10206 & 26,8 & 27,1 \\
\hline D. Araújo \& O. Peixoto 6527 & 26,7 & 26,8 \\
\hline \multicolumn{3}{|l|}{ T. phlomoides } \\
\hline C. Farney \& D. Araújo 3466 & 47,0 & 49,4 \\
\hline \multicolumn{3}{|l|}{ Thryallis brachystachys } \\
\hline Belém \& J.M. Mendes s.n.(RB1405 & 23) 30,3 & 31,0 \\
\hline Sampaio 7893 & 33,0 & 34,0 \\
\hline
\end{tabular}

\section{Discussão}

Byrsonima sericea foi a única espécie a apresentar grãos de pólen isopolares, tricolporados e exina granulada. A maioria dos grãos de pólen apolares apresentou forma subesferoidal, exceto Banisteriopsis sellowiana e Peixotoa hispidula que apresentaram forma cuboidal, colpóides nítidos ou não, associados ou não aos poros; a sexina foi descrita como rugulada na maioria das espécies sendo encontrada, ainda, sexina tectado-perfurada, microrreticulada e insulada; em sua grande maioria, a sexina foi mais espessa que a nexina, com exceção de Heteropterys alternifolia e Tetrapterys acutifolia, onde esta foi tão espessa quanto à nexina e em Banisteriopsis sellowiana, onde a sexina foi menos espessa que a nexina.

Erdtman (1952) descreveu os grãos de pólen de Stigmaphyllon ciliatum, Tetrapteris mexicana Hook \& Arn. e Thryallis angustifolia Benth. Os resultados encontrados pelo autor foram também, em parte, observados no presente trabalho. Em Thryallis angustifolia, Erdtman (1952) considerou os grãos de pólen como tricolporados, característica essa não corroborada aqui.

Salgado-Labouriau (1973) analisou os grãos de pólen de táxons encontrados nos cerrados brasileiros e, dentre eles, Banisteriopsis campestris (A. Juss.) Little, Byrsonima verbascifolia Rich. ex Juss., espécies de Camarea St. Hill. e Peixotoa reticulata St. Hill. A autora criou tipos polínicos subordinando espécies de gêneros diferentes nestes tipos. Assim, Salgado-Labouriau (1973) criou os tipos: Byrsonima verbascifolia, subordinando sob esse, Byrsonima verbascifolia Rich. ex Juss var. verbascifolia, Byrsonima verbascifolia subsp. discolor Need., Galphimia brasiliensis A. Juss. Sob o Tipo Camarea, foram reunidas as espécies Camarea affinis A.St. Hill., C. ericoides A. St. Hill. e C. triphylla A. Juss. O Tipo Peixotoa reticulata englobou as espécies Peixotoa reticulata e Banisteriopsis campestris. Os resultados aqui encontrados para as espécies Banisteriopsis sellowiana, Byrsonima sericea e Peixotoa hispidula não puderam ser enquadrados nos tipos acima citados, uma vez que o Tipo Byrsonima verbascifolia possui exina reticulada, o 

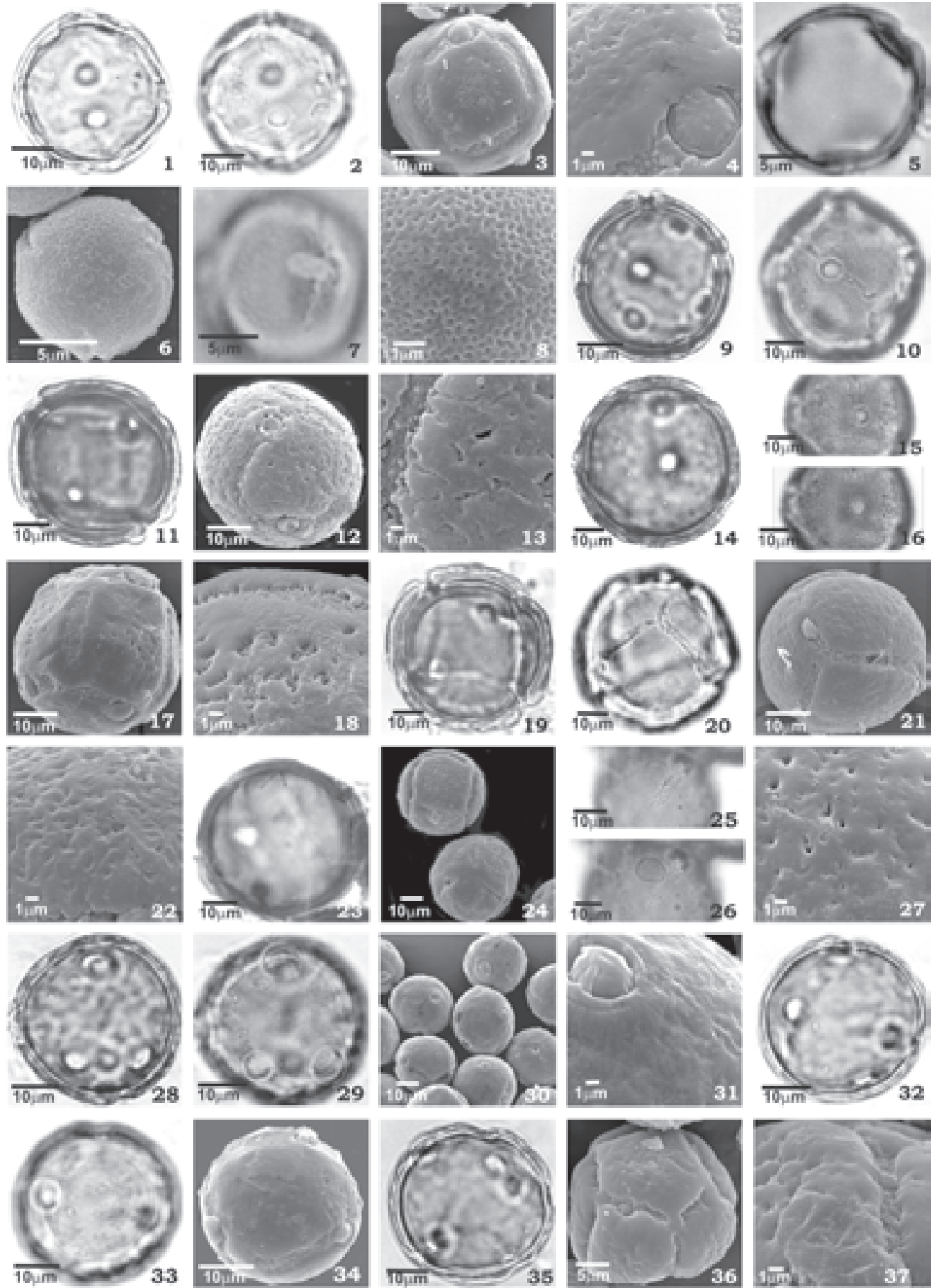

Figuras 1-37. Grãos de pólen de espécies de Malpighiaceae. Figuras 1-4. Banisteriopsis sellowiana. Vista geral: 1. Corte óptico. 2. Abertura e superfície, 3. Aspecto geral do grão de pólen (MEV). 4. Detalhe da superfície e abertura (MEV). 5-8. Byrsonima sericea. Vista polar: 5. Corte óptico. 6. Apocolpo. Vista equatorial. 7. Abertura. 8. Detalhe da superfície. 9-13. Heteropterys alternifolia. Vista geral: 9. Corte óptico. 10. Superfície. 11. Corte óptico. 12. Aspecto geral do grão de pólen (MEV). 13. Detalhe da superfície (MEV). 14-18. H. bicolor. Vista geral: 14. Corte óptico. 15-16. Análise de L.O. 17. Aspecto geral da superfície (MEV). 18. Detalhe da superfície (MEV). 19-22. H. chrysophylla. Vista geral: 19. Corte óptico. 20. Superfície. 21. Aspecto geral do grão de pólen (MEV). 22. Detalhe da superfície (MEV). 23-27. H. coleoptera. Vista geral: 23. Corte óptico. 24. Aspecto geral da superfície (MEV). 25-26. Análise de L.O. 27. Detalhe da superfície (MEV). 28-31. H. fluminensis. Vista geral: 28. Corte óptico. 29. Superfície e abertura 30. Aspecto geral do grão de pólen (MEV). 31. Detalhe da superfície e da abertura (MEV). 32-34. H. rufula. Vista geral: 32. Corte óptico. 33. Superfície. 34. Aspecto geral da superfície (MEV). 35-37. Hiraea cuneata. Vista geral: 35. Corte óptico. 36. Aspecto geral da superfície (MEV). 37. Detalhe da superfície (MEV). 

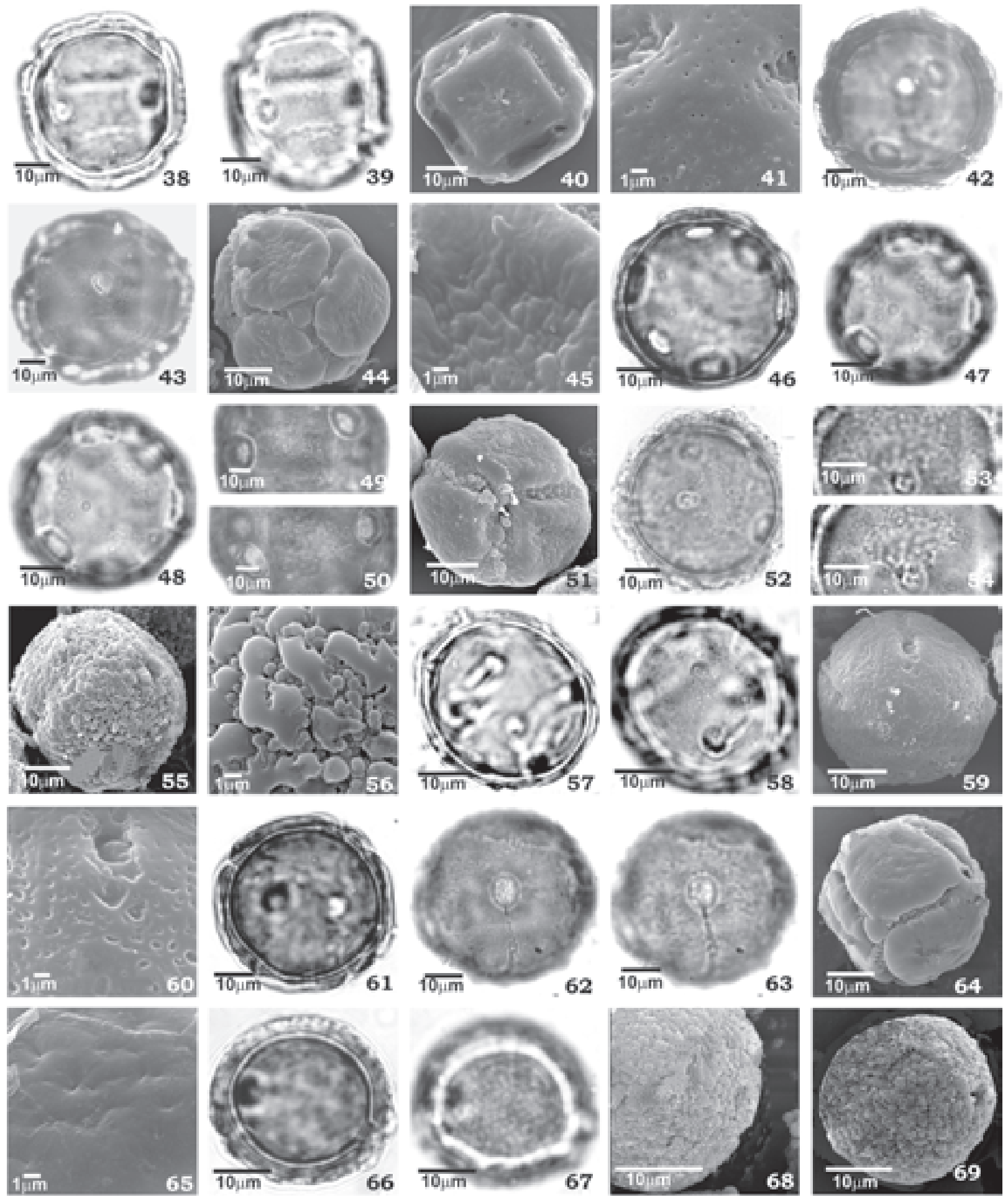

Figuras 38-69. Grãos de pólen de espécies de Malpighiaceae. Figuras 38-41. Peixotoa hispidula. Vista geral: 38. Corte óptico. 39. Superfície e abertura. 40. Aspecto geral da superfície (MEV). 41. Detalhe da superfície (MEV). 42-45. Stigmaphyllon arenicola. Vista geral: 42. Corte óptico. 43. Abertura. 44. Aspecto geral da superfície (MEV). 45. Detalhe da superfície (MEV). 46-47. S. auriculatum. Vista geral: 46. Corte óptico. 47. Abertura e superfície. 48-51. S. ciliatum. Vista geral: 48. Contorno. 49-50. Análise de L.O. 51. Aspecto geral da superfície (MEV). 52-56. S. paralias. Vista geral: 52. Corte óptico. 53-54. Análise de L.O. 55. Aspecto geral da superfície (MEV). 56. Detalhe da superfície (MEV). 57-60. Tetrapterys acutifolia. Vista geral: 57. Corte óptico. 58. Superfície e abertura. 59. Aspecto geral da superfície (MEV). 60. Detalhe da superfície (MEV). 61-65. T. phlomoides. Vista geral: 61. Corte óptico. 62-63. Análise de L.O. 64. Aspecto geral da superfície (MEV). 65. Detalhe da superfície (MEV). 66-69. Thryallis brachystachys. Vista geral: 66. Corte óptico. 67. Aspecto geral da superfície. 68. Detalhe da superfície (MEV). 69. Aspecto geral da superfície (MEV). 
Tipo Peixotoa reticulata foi descrito como possuindo 6-poros, exina espessa e escura, estratificação indistinta nos grãos de pólen acetolisados. Os grãos de pólen de Banisteriopsis sellowiana e Peixotoa hispidula aqui descritos apresentaram, respectivamente, 6 e 8 poros e as camadas da exina puderam ser medidas e a superfície, descrita.

Makino (1986) analisou os grãos de pólen de alguns gêneros de Malpighiaceae da flora da Reserva do Parque Estadual das Fontes do Ipiranga, São Paulo e, dentre eles, Banisteriopsis Robins. ex Small, Byrsonima Rich. ex A.L. Juss., Heteropterys Kunth, Hiraea Jacq. e Tetrapterys Cav. Os resultados encontrados pela autora foram aqui também observados para Byrsonima sericea com exceção da ornamentação da sexina que foi considerada pela autora como finamente reticulada e no presente estudo, como microrreticulada. Os resultados encontrados por Makino (1986) para Banisteriopsis diferiram daqueles aqui observados quando foram considerados o número de poros (4-5)-6pantoporados, a sexina granulada, com gemas na superfície dos colpóides, a sexina microrreticulada e a sexina menor que a nexina. Quanto ao gênero Heteropterys, os resultados aqui encontrados diferiram dos apresentados por Makino (1986) quando foi considerado o número de poros (de 3 a 12 poros), enquanto, no presente trabalho as espécies apresentaram 6-8 poros; a sexina, para a autora, variou, podendo ser lisa, microrreticulada ou reticulado-rugulada e a sexina, menos espessa que a nexina. No presente estudo, as espécies de Heteropterys apresentaram sexina e superfície do colpóide rugulados, sexina tectado-perfurada em $H$. coleoptera e escabrada com perfurações em $H$. fluminensis e $H$. rufula. Em relação às espécies de Tetrapterys, Makino (1986) obteve resultados que não foram encontrados aqui, quando foram comparadas as espessuras da sexina e sua relação com a nexina (em todas as espéc ies a sexina foi mais espessa do que a nexina). As características relativas à disposição dos colpóides, à posição do poro nos colpóides e à interrupção destes, não foram observadas no presente estudo.

Roubik \& Moreno (1991) estudaram 20 espécies pertencentes a 10 gêneros de Malpighiaceae ocorrentes na Ilha Barro Colorado, Panamá. Dentre os gêneros ou espécies analisadas por esses autores, estão Banisteriopsis, Byrsonima, Hiraea, Stigmaphyllon e Tetrapterys. A ornamentação do grão de pólen das espécies pertencentes a esses gêneros, segundo esses autores, variou de psilada, escabrada, verrugada, reticulada até foveolada. No presente estudo a ornamentação dos grãos de pólen das espécies destes gêneros foi descrita como rugulada, na maioria, Tetrapteris acutifolia e Byrsonima sericea foram às únicas a apresentar sexina microrreticulada.

Com base nos resultados obtidos na análise dos grãos de pólen de espécies de Malpighiaceae, podese estabelecer que:

- Byrsonima sericea apresentou grãos de pólen totalmente diferentes daqueles dos outros gêneros aqui estudados, principalmente, por serem pequenos, isopolares e tricolporados.

- As demais espécies dos outros gêneros (Banisteriopsis sellowiana, Heteropterys, Hiraea cuneata, Peixotoa hispidula, Stigmaphyllon, Tetrapterys e Thryallis brachystachys) apresentaram atributos que puderam ser utilizados na chave polínica para separá-las. As principais características utilizadas foram: tamanho dos grãos de pólen, quantidade e dimensões dos poros, a ornamentação da sexina e dos colpóides.

Assim, pode-se concluir, que as espécies de Malpighiaceae aqui analisadas são, palinologicamente, distintas e as características polínicas mostraram ser um bom recurso disponível aos pequisadores que poderão utilizar na taxonomia, na Aeropalinologia, na Melissopalinologia e demais áreas afins.

\section{Agradecimentos}

Ao Laboratório de Ultraestrutura Celular, do Instituto de Biofísica da Universidade Federal do Rio de Janeiro (UFRJ), na pessoa da técnica de microscopia eletrônica de varredura, Nôemia Rodrigues Gonçalves. Ao CNPq pela bolsa concedida à primeira autora.

\section{Literatura citada}

Aguiar, C.M.L. \& De-Almeida, G.F. 2002. Atividade diária de coleta de óleo por espécies de Centris (Hymenoptera: Apidae) em Mcvaughia bahiana W.R. Anderson (Malpighiaceae) na Caatinga. Acta Biológica Leopoldensia 24: 131-140.

Alves, G.L. \& Franco, M.R.B. 2003. Headspace gas chromatography-mass spectrometry of volatile compounds in murici (Byrsonima crassifolia L. Rich). Journal of Chromatography 985: 297-301. 
APG. 2003. An update of the Angiosperm Phylogeny Group classification for the orders and families of flowering plants: APG II. Botanical Journal of the Linnean Society 141:399- 436.

Barth, O.M. \& Melhem, T.S. 1988. Glossário Ilustrado de Palinologia. Ed. UNICAMP, Campinas.

Barth, O.M. 1989. O pólen no mel brasileiro. Gráfica Luxor, Rio de Janeiro.

Erdtman, G. 1952. Pollen Morphology and Plant Taxonomy - Angiosperms. Almqvisit \& Wiksell, Stockholm.

Lombello, R.A. \& Forni, M.E.R. 2001. Cytological studies on Banisteriopis C.B. Robison ex Small and Heteropterys Kunth (Malpighiaceae). Cytologia - Tokio $66: 253-259$.

Makino, W.H. 1986. Flora polínica da Reserva do Parque Estadual das Fontes do Ipiranga - Família 125-Malpighiaceae. Hoehnea 13: 21-30.

Melhem, T.S., Cruz-Barros, M.A.V., Corrêa, A.M.S., Makino-Watanabe, H., Silvestre-Capelato, M.S.F. \& Gonçalves-Esteves, V. 2003. Variabilidade polínica em plantas de Campos de Jordão (São Paulo, Brasil). Boletim do Instituto de Botânica 16: 9-104.
Melo, M.M., Vasconcelos, A.C., Dantas, G.C., Serakides, R. \& Alzamora, F.F. 2001. Experimental intoxication of pregnant goats with Tetrapterys multiglandulosa A. Juss. (Malpighiaceae). Arquivo Brasileiro de Medicina Veterinaria e Zootecnia 53: 58-65.

Punt, W., Blackmore, S., Nilsson, S. \& Le Thomas, A. 2007. Glossary of pollen and spore terminology. Review of Paleobotany and Palynology 143: 1-81.

Roubik, D.W. \& Moreno, J.E. 1991. Pollen and Spores of Barro Colorado Island. Missouri Botanical Garden, New York.

Salgado-Labouriau, M.L. 1973. Contribuição à Palinologia dos Cerrados. Academia Brasileira de Ciências, Rio de Janeiro.

Salgado-Labouriau, M.L., Vanzolini, P.E. \& Melhem, T.S. 1965. Variation of polar axes and equatorial diameters in pollen grains of two species of Cassia. Grana palynologica 6: 166-176.

Schwarz, M.J., Houghton, P.J., Rose, S., Jenner, P. \& Lees, A.D. 2003. Activities of extract and constituents of Banisteriopsis caapi relevant to parkinsonism. Pharmacology Biochemistry and Behavior 75: 627-633. 